\title{
Joint production, setup and preventive maintenance policies of unreliable two-product manufacturing systems
}

\author{
M. Assid ${ }^{a}$, A. Gharbi ${ }^{a^{*}}$, A. Hajji ${ }^{b}$ \\ a Automated Production Engineering Department, École de technologie supérieure, \\ Production System Design and Control Laboratory, University of Québec, 1100 Notre Dame \\ Street West, Montreal, QC, Canada H3C 1K3 \\ ${ }^{\mathrm{b}}$ Department of Operations and Decision Systems \& CIRRELT. Laval University. Pavillon \\ Palasis-Prince 2325, rue de la Terrasse Québec (Québec) G1V 0A6
}

\begin{abstract}
This article addresses the problem of joint optimization of production, setup and maintenance activities of unreliable manufacturing system producing two products. Given the complexity of the problem in a dynamic and stochastic environment, the literature has treated the problem separately by considering each axis individually (setup, production and maintenance) or by combining two axes simultaneously (production-setup; production-maintenance). Following the trend of scientific research advances that supports the fact that an integrated control leads to best performances, the main objective of this paper is to provide a control policy that will simultaneously combine the production, the setup and the preventive maintenance activities. To tackle the problem, an experimental resolution approach using combined continuous/discrete event simulation models is considered. The aim is to accurately imitate the production system behavior and to optimize the control policy parameters which minimize the total cost incurred. An in-depth study of the effects of the system parameters variation on the performance of the studied policies is performed in order to draw meaningful conclusions and to illustrate the robustness of the proposed resolution approach.
\end{abstract}

Keywords: Production / setup control, preventive maintenance, inflexible and unreliable manufacturing system, simulation modeling, optimization, response surface methodology.

\section{Introduction}

In the context of unreliable manufacturing system, one of the most effective strategies is the hedging point policy (HPP) concept (Akella and Kumar, 1986). It involves the building of an 
appropriate safety stock while the machine is operational to respond to customer demands when the machine is down. For failure and repair times described by homogeneous Markov processes, the optimality of the HPP is proved in the case of one-machine one-product type manufacturing system $\left(\mathrm{M}_{1} \mathrm{P}_{1}\right)$ with constant demand rate. Many subsequent works have extended the concept of HPP to other manufacturing systems. For example, Kenné and Gharbi (2000) considered random demands and general failure and repair time distributions and Gharbi et al. (2011) developed the Multiple HPP (MHPP) for multiple state systems producing one product.

Integrating preventive maintenance (PM) in the production planning has also attracted many researchers since machine breakdowns may disturb production process and cause delay in schedules. The PM function, in the overall manufacturing sector, is a priority and is central to the concerns of manufacturers. Its role is crucial and is manifested in the failures prevention and the maintaining of production tools in service. Several integrated models that coordinate PM planning decisions with the production scheduling were proposed (Cassady and Kutanoglu, 2005; Wang and Liu, 2013). The main objective was to minimize the total expected weighted completion time of jobs. Other studies argued the interests to integrate PM and planning for production as PM helps maintain the production tool in service and improve the system performances (Wang, 2002). Wee and Widyadana (2013) explain that PM can result in savings due to an increase of the effective service life of the system. Reineke et al. (1999) addressed the problem of determining the appropriate age PM policy in order to improve the availability and cost performance for a system that has high availability requirements. For a single machine manufacturing system, Kuo and Chang (2007) developed an integrated maintenance scheduling and production planning optimization model with the objective of minimizing the total tardiness. Aramon Bajestani et al. (2014) formulated a Markov decision process model for the problem of integrated maintenance and production scheduling in a deteriorating multi-machine production system. The computational results achieved by integer programming show that exploiting online condition monitoring information in maintenance and production decisions leads to significant cost savings compared to a greedy heuristic. Another significant branch of the literature, which includes our work, is rather interested in the dynamics of the manufacturing system (inventories levels and machine states), with the aim of optimizing production rates and maintenance intervals. Indeed, despite their benefits, PM actions as well as setup operations entail the downtime of production units which can lead to shortages. The combined production/maintenance cost is 
used as the performance criterion because of its major importance in improving the competitiveness of companies.

For single-product manufacturing systems, mathematical models have been developed in order to combine PM strategies with production control policies (Rezg et al., 2008; Ayed et al., 2012), but many do not arise from an optimal structure. Gharbi and Kenné (2000) proposed a near-optimal age-dependent control policy in the case of an increasing failure rate. PM interventions are then planned at failure or according to the Age Replacement Policy (ARP) concept. Assuming that PM reduces the failure frequencies, Gharbi et al. (2007) developed joint PM and production policies depending on produced part inventory levels. Berthaut et al. (2011) compared the effect of cost and time parameters of the system on PM strategies when combined with the HPP. They considered three PM strategies: the ARP, the Block Replacement Policy (BRP) and the Modified Block Replacement Policy (MBRP). Contrary to what researchers have been shown in the literature, the MBRP outperforms the ARP in terms of incurred total cost which is composed of both inventory and maintenance costs. Indeed, the proposed control policy can skip PM actions if the time elapsed since the last maintenance action is lower than a given threshold. This will reduce the risk of shortages and the waste of new components since it avoids consecutive failures and PM. In the case of a reliable $\mathrm{M}_{1} \mathrm{P}_{1}$ subject to quality deterioration, Dhouib et al. (2012) developed a joint inventory control and an age-based PM policy in order to reduce the switch rate to the out of control state for a $\mathrm{M}_{1} \mathrm{P}_{1}$ incorporating imperfect production process. In the case of an unreliable $\mathrm{M}_{1} \mathrm{P}_{1}$ subject to quality and reliability deterioration, Rivera-Gomez et al. (2013) proposed a control policy that simultaneously determines the optimal production plan and PM strategies. They utilize PM interventions with the objective of reducing the level of deterioration and improving the quality of the units produced.

For systems with multiple product types, few authors addressed the influence of nonnegligible setup time and cost on the system performance. Bai and Elhafsi (1997) propose a suitable production and setup control policy called the Hedging Corridor Policy (HCP). It guides the surplus trajectory to target buffer stock thresholds to hedge against future capacity shortages caused by machine failures and large setup times. In the same context, Gharbi et al. (2006) develop the Modified Hedging Corridor Policy (MHCP) in order to effectively determine a better sequence of setups and further reduce the risk of shortage. An in-depth study was conducted in (Assid et al., 2014b) addressing implementation issues between the 
HCP and the MHCP via quantitative and qualitative criteria. Hajji et al. (2009) studied production and changeover control production for a buffered flow-shop producing several types of parts. They developed a state-dependent HPP including feedbacks on the raw material and finished products inventories.

The problem of optimisation of production, setup and maintenance activities of unreliable manufacturing systems producing different products has been treated separately as detailed in preceding paragraphs. To the best of our knowledge, no work has addressed the optimal control problem while simultaneously considering production and PM planning in the case of two-product non-flexible manufacturing systems where both setup and maintenance interventions have non-negligible durations. The main objective of this work is to propose a proper joint control policy structure which combines production, setup and PM activities in an unreliable manufacturing environment producing two products. Our work addressed how production and setup control policies can be adjusted to take into account PM strategies in an accurate and timely manner, so as to minimize the total cost of inventory/backlog, setup, repair and PM costs. PM is used to reduce the possible harmful effects of setup operations and the machine breakdown which is characterized by an increasing failure rate, and therefore to achieve further cost savings and more availability of the production machine. The rest of this paper is organized as follows. In Section 2, the manufacturing system and the studied control policies are described. The joint production/setup/maintenance control problem of the system is also discussed. Section 3 summarizes the system data, the numerical example and the experimental resolution approach used in order to determine the optimal control parameters. A sensitivity analysis is conducted in Section 4. Section 5 presents deep analysis aiming to show the influence of many system variables on the optimal control parameters and a comparative study of the total cost incurred by all policies considered. The potential economic advantage of the production and setup planning versus the PM actions is also discussed on several scenarios. This article closes in Section 6 with a conclusion.

\section{System description and problem definition}

In order to illustrate the integration of PM strategies with production and setup control policies as presented above, the manufacturing system considered consists of a non-flexible machine capable of producing two products. Each product should be stored in a given area before being delivered to customers. The machine is subject to random repairs and 
breakdowns which are defined by non-exponential distributions and may cause stock-outs. Preventive interventions are also planned in order to increase the production system availability. In addition, the cost and the time required to switch the production from one product type to another are considered non-negligible. The overall structure of the manufacturing system is illustrated in Figure 1.

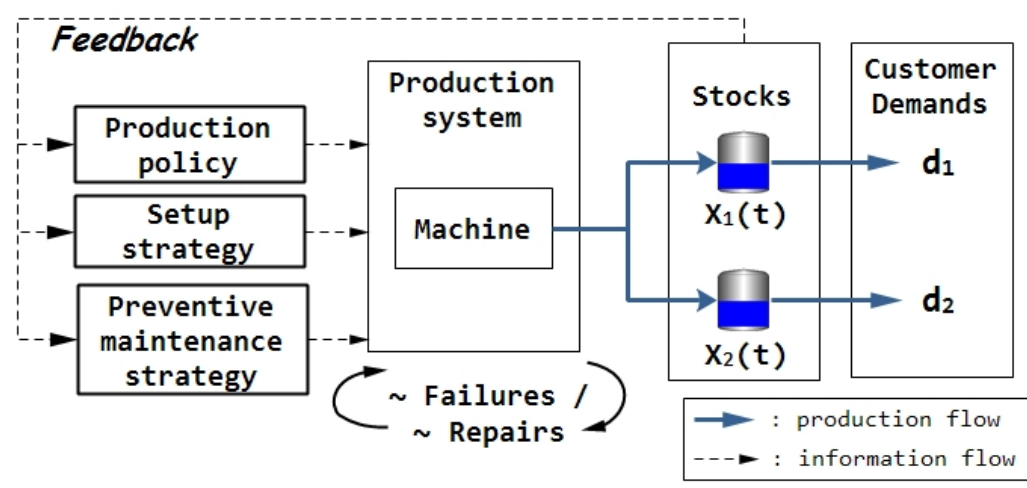

Figure 1. Structure of the manufacturing system under study.

\subsection{Notation}

The following notations are used throughout the paper. $\forall \mathrm{i}, \mathrm{j} \in \mathrm{I}^{2}=\{1,2\}^{2}$

$\mathrm{P}_{\mathrm{i}} \quad$ : Type of product $\mathrm{i}$

$\mathrm{x}_{\mathrm{i}}(\mathrm{t}) \quad$ : Inventory level (or backlog) of product $\mathrm{P}_{\mathrm{i}}$ at time $\mathrm{t}$

$\mathrm{d}_{\mathrm{i}} \quad$ : Constant demand rate of the product $\mathrm{P}_{\mathrm{i}}$ (product/time unit)

$\mathrm{u}_{\mathrm{i}}(\mathrm{t}) \quad$ : Production rate of the product $\mathrm{P}_{\mathrm{i}}$ at time $\mathrm{t}$ (product /time unit)

$\mathrm{U}_{\mathrm{i}}^{\max }$ : Maximum production rate of the product $\mathrm{P}_{\mathrm{i}}$ (product/time unit)

$\mathrm{Z}_{\mathrm{i}}^{\text {inv }} \quad$ : Storage space capacity of the product $\mathrm{P}_{\mathrm{i}}$

$\mathrm{Z}_{\mathrm{PM}} \quad$ : Preventive maintenance threshold

$a_{i} \quad$ : Setup threshold of the product $P_{i}$

$\mathrm{T}_{\mathrm{PM}} \quad$ : Time interval between two preventive maintenance interventions

$T_{i j}^{S} \quad$ : Setup time required to switch from the production of $P_{i}$ to $P_{j}, i \neq j$ (time unit)

$\mathrm{c}_{\mathrm{i}}^{+} \quad: \mathrm{P}_{\mathrm{i}}$ inventory cost $(\$ /$ product/time unit)

$\mathrm{c}_{\mathrm{i}}^{-} \quad: \mathrm{P}_{\mathrm{i}}$ backlog cost (\$/product/time unit)

$c_{i j}^{S} \quad$ : Setup cost to switch from the production of $P_{i}$ to $P_{j}, i \neq j$ (\$/Setup action)

$\mathrm{c}_{\mathrm{pm}} \quad$ : Preventive maintenance (PM) cost (\$/PM action) 
$\mathrm{c}_{\mathrm{cm}} \quad$ : Corrective maintenance $(\mathrm{CM}) \operatorname{cost}(\$ / \mathrm{CM}$ action)

$\mathrm{C}_{\mathrm{p}}^{*} \quad$ : Optimal total cost incurred of the control policy $\mathrm{p}$

\subsection{Problem definition}

The state of the system is modeled by two components. On one hand, the cumulative inventory of both products $\mathrm{X}(\mathrm{t})=\left(\mathrm{x}_{1}(\mathrm{t}), \mathrm{x}_{2}(\mathrm{t})\right) \in \mathrm{R}^{2}$ which corresponds to a continuous variable in time and on the other hand, the discrete state of the machine $\zeta(t)$. The dynamics of the production system which consists of a single machine, takes the value 1 if the machine $M$ is operational, 2 if it fails and 3 if it is subject to preventive maintenance actions. Thus, the stochastic and dynamic system behavior is described by the state variables $(X(t), \zeta(t))$ with $\mathrm{X}(\mathrm{t})=\left(\mathrm{x}_{1}(\mathrm{t}), \mathrm{x}_{2}(\mathrm{t})\right) \in \mathrm{R}^{2}$ and $\zeta(\mathrm{t}) \in \mathrm{M}=\{1,2,3\}$.

$$
\zeta(t)=\left\{\begin{array}{l}
1: \text { if } M \text { is operational } \\
2: \text { if } M \text { is down }(C M) \\
3: \text { if } M \text { is subject to PM actions }
\end{array}\right.
$$

The following differential equation represents the stocks dynamic $\dot{X}(t)=\left(\dot{x_{1}}(t), \dot{x_{2}}(t)\right) \in R^{2}$. It is expressed in terms of the production rate $u_{i}(t), i \in\{1,2\}$ and the customer demand $d_{i}$.

$$
\begin{cases}\dot{x_{1}}(t)=u_{1}(t)-d_{1}, & x_{1}(0)=x_{1} \\ \dot{x_{2}}(t)=u_{2}(t)-d_{2}, & x_{2}(0)=x_{2}\end{cases}
$$

Where $x_{1}$ and $x_{2}$ refer respectively to the initial inventory level of both products $P_{1}$ and $P_{2}$. The following production capacity constraint must be satisfied at all times:

$$
0 \leq \mathrm{u}_{\mathrm{i}}(\mathrm{t}) \leq \mathrm{U}_{\mathrm{i}}^{\max } * \mathrm{I}(\zeta(\mathrm{t})=1), \quad \forall \mathrm{i} \in\{1,2\}
$$

With,

$$
\mathrm{I}(\zeta(\mathrm{t})=\delta)= \begin{cases}1 & \text { if } \zeta(\mathrm{t})=\delta \\ 0 & \text { otherwise }\end{cases}
$$

Regarding the transition probabilities of our stochastic process, except the transition from state 1 to 3 which is in our case function of the PM decision variable (see next paragraph), the 
other transition probabilities are constant and represent a function of the parameters of a given probability distribution (see section 3.3 for more details).

Two parameters are used in order to model setup operations: the time $\tau_{\mathrm{k}}$ at which the $\mathrm{k}^{\text {th }}$ $(\mathrm{k} \in \mathrm{N})$ setup action begins and the pair $\mathrm{i}_{\mathrm{k}} \mathrm{j}_{\mathrm{k}+1}$ which represents the production transition from the product $P_{i}$ to the product $P_{j}, i \neq j$. The sequence of setup actions $\varphi$ is then defined by $\varphi=\left\{\left(\tau_{0}, \mathrm{i}_{0} \mathrm{i}_{1}\right),\left(\tau_{1}, \mathrm{i}_{1} \mathrm{i}_{2}\right), \ldots\right\}$. Therefore, the decision variables of the control policy, which combines the production, the setup and the maintenance planning, are the production rate $u_{i}(t), i \in\{1,2\}$, the sequence of setups $\varphi$ and the time interval between two PM interventions $\mathrm{T}_{\mathrm{PM}}$. The objective of the stochastic optimal control problem is to optimize the system control parameters which minimize the total cost function J(. ) over an infinite horizon.

$$
J\left(X, \zeta, u_{1}, u_{2}, \varphi, T_{P M}\right)=E\left[\int_{0}^{\infty} e^{-\rho t} g(.) d t+\sum_{j=0}^{\infty} e^{-\rho \tau_{j}} C_{i_{j} i_{j+1}}^{s}\right]
$$

With, $\rho$ is the discount rate, $g($.$) is the function of the instantaneous inventory, backlog and$ maintenance cost, it is defined as:

$$
g(X, \zeta)=c_{i}^{+} x_{i}^{+}(t)+c_{i}^{-} x_{i}^{-}(t)+c_{c m} E_{c m}^{+}(t)+c_{p m} E_{p m}^{+}(t)
$$

Where,

$$
\left\{\begin{array}{l}
x_{i}^{+}=\max \left(0, x_{i}\right) \\
x_{i}^{-}=\max \left(-x_{i}, 0\right)
\end{array}\right.
$$

And,

$$
E_{c m}^{+}(t)=\left\{\begin{array}{ll}
1 & \text { if } \zeta(t)=2 \\
0 & \text { otherwise }
\end{array} \quad E_{p m}^{+}(t)= \begin{cases}1 & \text { if } \zeta(t)=3 \\
0 & \text { otherwise }\end{cases}\right.
$$

The expected total cost (3) is composed of two terms. The first one consists on the discounted cost $\mathrm{g}($.$) over an infinite horizon. Given that the calculation of \mathrm{g}($.$) occurs on a continuous$ basis, the actual values of the incurred instantaneous costs are summed as follows: $\int_{0}^{\infty} \mathrm{e}^{-\rho t} \mathrm{~g}(.) \mathrm{dt}$. The second one consists on the actual values of the setup costs incurred in a discrete basis at times $\tau_{\mathrm{j}}$. These costs are simply actualised and summed over the infinite 
horizon. The value function of the optimization problem is described by the following function:

$$
\mathrm{v}(.)=\inf _{\left(\mathrm{U}, \varphi, \mathrm{T}_{\mathrm{PM}}\right) \in \Gamma(\alpha)} \mathrm{J}\left(\mathrm{X}, \zeta, \mathrm{u}_{1}, \mathrm{u}_{2}, \varphi, \mathrm{T}_{\mathrm{PM}}\right) ; \forall \mathrm{X} \in \mathrm{R}^{2}, \zeta \in\{1,2,3\}
$$

The only way to find an integrated steady state policy to control production, setup and maintenance actions as a function of the state of the system is to solve the Hamilton-Jacobi Bellman (HJB) equations associated to the value function (5). An analytical solution in this context is simply impossible given the difficulty to calculate the value function of the problem. In the case of production and setup planning, both works of Gharbi et al. (2006) and Bai and Elhafsi (1997) solved numerically the HJB equation of the joint production and setup problem. However, when the PM interval decisions are integrated as in our case, the state of the system become larger and the numerical solution of the problem more difficult. Given the complexity of our control problem, we are based on the literature which has treated the problem separately by considering each axis (production-setup; production-maintenance) individually.

Two of the most complete production and setup control policies are adopted in this work: the HCP the MHCP which has been improved in this work in order to reduce the risk of shortages. These control policies are respectively proposed by Bai and Elhafsi (1997) and Gharbi et al. (2006) whom discussed the interaction between production activities and setup actions for $\mathrm{M}_{1} \mathrm{P}_{2}$ manufacturing system with significant setup times and costs. Two PM strategies are integrated to the aforementioned production and setup policies. In addition to the BRP which is easier to manage than the ARP in an industrial context, the second PM strategy considered is based on the BRP but takes into account the inventory level of each product before coming to a decision on the execution of PM actions. This condition is inspired by the MBRP used in (Berthaut et al., 2011) and which can skip PM interventions if $\mathrm{CM}$ actions are recently performed. This not only protects the system against higher shortages, but it also reduces the PM cost.

\subsection{Description of the considered control policies}

Both complete production and setup control policies and the two PM strategies adopted in this work (see section 1) are defined as follows: 


\section{- $\quad$ Classical Hedging Corridor Policy (HCP):}

The Hedging Corridor Policy (HCP) is characterised by a single control parameter $\mathrm{Z}_{\mathrm{i}}^{\text {inv }}$, $i \in\{1,2\}$ for each product $\mathrm{P}_{\mathrm{i}}$. The machine operates at maximum capacity throughout the availability period so that as soon as the inventory level $\mathrm{x}_{\mathrm{i}}$ of the product being manufactured reaches its threshold $\mathrm{Z}_{i}^{\text {inv }}$, a setup action is performed in order to switch the production to the other product.

- $\quad$ Improved Modified hedging corridor policy (IMHCP)

Our Improved Modified Hedging Corridor Policy (IMHCP) improves the MHCP proposed in (Gharbi et al., 2006) by conducting setups before the stocks of a product type reaches the zero value and then reducing the risk of shortages. Unlike the MHCP, a new parameter $b_{i}>0$ ( $\mathrm{i} \in\{1,2\})$ for each product is introduced. It represents the inventory level needed to perform setup operations before shortages. The main advantage of IMHCP compared to HCP is still to reduce the number of setups since no setup operation is performed unless a risk that a product type will be out of stock is observed. Six control parameters $Z_{i}, a_{i}$ and $b_{i}$, $(i \in\{1,2\}$ with $a_{i} \leq Z_{i}$ ) characterize the IMHCP. When a product inventory level $x_{i}$ exceeds the $a_{i}$ value, $a$ setup operation is performed only if the inventory level of the other product $x_{j}(j \in\{1,2\}$ and $\mathrm{j} \neq \mathrm{i})$ is less than the parameter $b_{j}\left(b_{j}>0, i \in\{1,2\}\right)$. Note that when $x_{i}$ reaches its threshold $\mathrm{Z}_{\mathrm{i}}$, the production rate is adapted to the demand rate.

- $\quad$ Classical Block Replacement Policy (B)

The first PM strategy integrated to the aforementioned production and setup policies is the Block Replacement Policy “B”. It is characterized by preventive replacements which are held and executed periodically at predetermined time intervals $\mathrm{kT}_{\mathrm{PM}}(\mathrm{k}=1,2, \ldots)$ regardless of the machine age.

- $\quad$ Block Replacement Policy with jumps according to the inventory level (BJ) 
In order to reduce the risk of shortages and the PM cost, the execution of PM actions on the production machine may depend on its age or on the utilization time from the last maintenance action (Berthaut et al., 2011). As the improvements made in the IMHCP, PM decisions could take into account the level of buffer stocks that would reduce the risk of shortages. However, our manufacturing system consists of a non-flexible machine capable of producing two products. This means that when it is restoring the buffer stock of a product, the inventory level of the other decrease while satisfying the customer demand. Similarly, the interruption of production activities during corrective and preventive maintenance or setup operations decreases the inventory level of both products. Therefore, our joint control policy will consider the amount of both inventories at a time before performing any PM action. This amount is expressed by the sum of both instantaneous inventories $\left(\mathrm{x}_{1}(\mathrm{t})+\mathrm{x}_{2}(\mathrm{t})\right)$ and governs the control of preventive interventions. In summary, the proposed "BJ" is characterized by $\mathrm{PM}$ interventions which are scheduled periodically at predetermined time intervals $\mathrm{kT}_{\mathrm{PM}}$ $(\mathrm{k}=1,2, \ldots)$, but they are performed only if the sum of both instantaneous inventories exceeds the threshold $\mathrm{Z}_{\mathrm{PM}}$ as follows:

$$
\left\{\begin{array}{l}
\mathrm{x}_{1}(\mathrm{t})+\mathrm{x}_{2}(\mathrm{t}) \geq \mathrm{Z}_{\mathrm{PM}} \\
\zeta(\mathrm{t})=1 \text { (i. e. the machine is operational) }
\end{array}\right.
$$

The four combined control policies considered in this paper are: HCP_B; IMHCP_B; HCP_BJ and IMHCP_BJ. A graphical representation of the variation of the instantaneous inventory levels $\left(\mathrm{x}_{1}(\mathrm{t})\right.$ and $\left.\mathrm{x}_{2}(\mathrm{t})\right)$ and the sum of both $\left(\mathrm{x}_{1}(\mathrm{t})+\mathrm{x}_{2}(\mathrm{t})\right)$ for the combined control policy IMHCP_BJ is presented (Figure 2) in order to further explain the operation of the system. Figure 2 shows that when the inventory level of the product being produced $\mathrm{x}_{\mathrm{i}}(\mathrm{t})$ ( $\mathrm{i} \in\{1,2\})$ is higher than the setup threshold $\mathrm{a}_{\mathrm{i}}\left(\mathrm{x}_{\mathrm{i}}(\mathrm{t})>\mathrm{a}_{\mathrm{i}}\right)$, the setup actions are performed only when the inventory level of the other product $x_{j}(t)(j \neq i)$ is less than the parameter $b_{j}$ $\left(\mathrm{x}_{\mathrm{j}}(\mathrm{t})<\mathrm{b}_{\mathrm{j}}\right)$ (1). Otherwise, the production rate is adapted to the demand rate as soon as $\mathrm{x}_{\mathrm{i}}(\mathrm{t})$ reaches the threshold $\mathrm{Z}_{\mathrm{i}}$ (2). Figure 2 shows also that when the machine is producing a product at the demand rate, the inventory level of the other product decreases while satisfying its customer demand. This involves the reduction of the inventories sum $\left(x_{1}(t)+x_{2}(t)\right)$ (3). Note that during the setup actions, the machine is stopped, hence the decrease in the amount of the sum of both inventories at a rate of $\mathrm{d}_{1}+\mathrm{d}_{2}$ (4). Similarly, when the machine is down, the inventory level of both products decreases until the end of repairs (6). Furthermore, preventive 
replacements are performed at fixed time intervals $\mathrm{kT}_{\mathrm{PM}}, \mathrm{T}_{\mathrm{PM}}=100$ with $\mathrm{k}=1,2, \ldots$ (5) However, they are canceled if $\mathrm{x}_{1}(\mathrm{t})+\mathrm{x}_{2}(\mathrm{t}) \leq \mathrm{Z}_{\mathrm{PM}}$ (7).
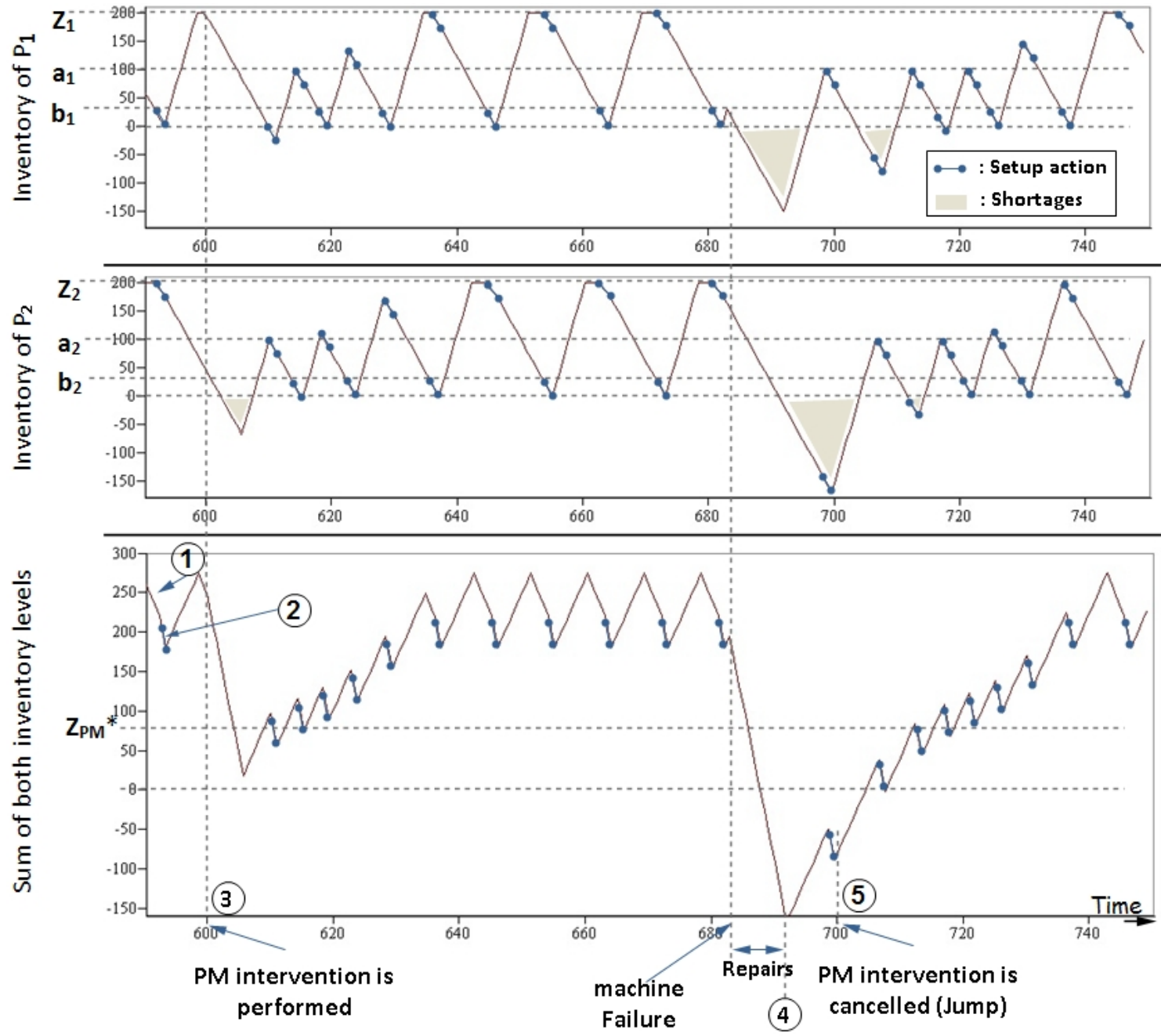

Figure 2. Variation of the inventories for the IMHCP_BJ $\left(Z_{1}=Z_{2}=200, b_{1}=b_{2}=40, a_{1}=a_{2}=100\right.$, $\mathrm{Z}_{\mathrm{PM}}=80$ and $\left.\mathrm{T}_{\mathrm{PM}}=100\right)$.

\section{Solution approach}

In this section, an experimental approach inspired by Gharbi et al. (2011) is used to determine experimentally the optimal control parameters which minimize the total incurred cost for each of the four control policies. This approach integrates simulation, design of experiments and response surface methodology. A block diagram of the resulting solution approach is depicted in Figure 3. The problem of the flow control for the considered manufacturing system is mathematically represented in the Control Problem definition block (1). The objective is to describe the dynamics of the system in terms of its states and the expression of the average 
total cost. This will help to define the decision variables, the constraints and the objective function to minimize. Such variables and parameters will help to develop the control policies. Based on block (1) and the literature, four joint production, setup and PM control policies are proposed (block (2)). Each one is parameterised by different control factors: $Z_{i}, a_{i}, b_{i}, T_{P M}$ and $\mathrm{Z}_{\mathrm{PM}}(\mathrm{i} \in\{1,2\})$ which affect the value the related output (the incurred total cost). Using the control policies as an input, the Continuous/Discrete event Simulation Model block (4) aims to conduct the experiments and to evaluate the production system performance. The cost related to each entry is defined as the output (response variable) of the simulation model. The Experimental design block (5) defines how the control factors can be varied in order to determine their effects and the effects of their interactions (i.e. ANOVA) on the response variable through a minimal set of simulation experiments. Subsequently, the response surface methodology represented by the block (6) is used to obtain the relationship between the significant main factors and interactions given in the previous step and the response variable. From this estimated relation, the optimal values of the control factors $\left(\mathrm{Z}_{i}^{*}, \mathrm{a}_{i}^{*}, \mathrm{~b}_{i}^{*}, \mathrm{~T}_{P M}^{*}\right.$ and $\mathrm{Z}_{P M}^{*}$ ) which minimize the total cost incurred are determined for each joint control policy (block (7)). The best joint control policy (block (8)) in terms of costs is then derived (see Sections 3.3, 4 and 5).

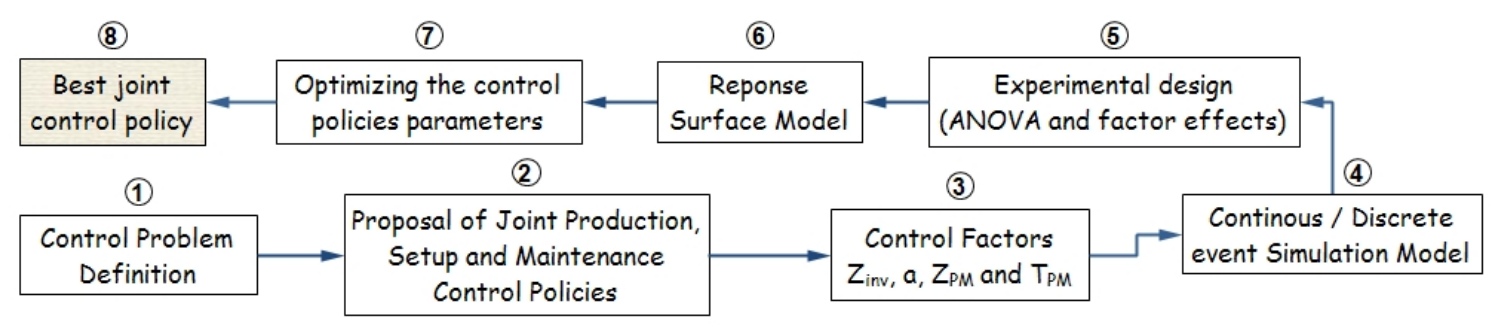

Figure 3. Proposed solution approach.

\subsection{Simulation models}

The Figure 4 shows the block-diagram schema of four combined discrete-continuous simulation models which represent the considered joint control policies (see Section 2.3). The choice of this modeling approach using the Arena "Flow Process" module is well known for its ability to greatly shorten the execution time when compared to the purely discrete models (Assid et al., 2014a). The continuous part essentially covers the calculation of the instantaneous inventories level, while the discrete part models the setup and the maintenance activities. 


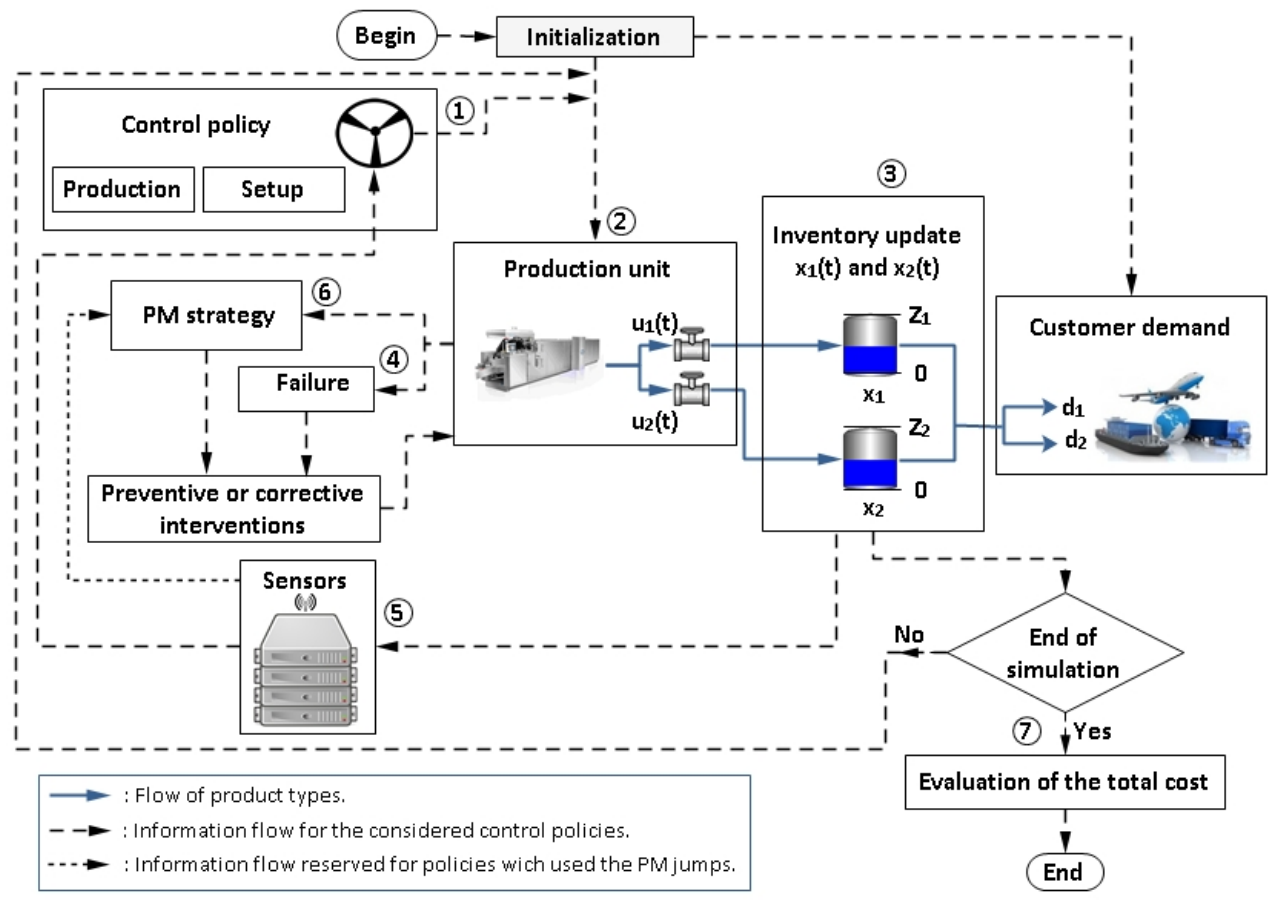

Figure 4. Simulation model diagram.

The simulation models structure consists of several networks: each must fulfil specific tasks such as the management of production activities, failures and repair interventions, etc (see Figure 4). They start by initializing the model variables such as the demand rate, the production capacity of the machine, the warm-up period, etc. Each simulation model depends on the considered control policy (Section 2.3) which represented in block (1), and then determines the product type to be manufactured and its production rate (block (2)) according to the value of inventory levels of both product types (block (3) and the system state (block (4)). Note that the failure detection is instantaneous and all maintenance interventions restore the machine to an as good as new condition. Our simulation models use a set of sensors (block (5) in order to continuously monitor both inventories and to check the availability of stocks. Thus a signal is sent whenever a threshold $\left(Z_{i}^{i n v}, a_{i}\right.$ and $\left.b_{i}, i \in\{1,2\}\right)$ is crossed. Depending on these instant data provided by the sensors (block (5)) as well as the production system state and the considered PM strategy, the PM strategy block (6) decides whether or not PM interventions will be performed: when the "BJ" is used, the PM interventions are performed only when the buffer stocks level is sufficient $\left(x_{1}(t)+x_{2}(t) \geq Z_{P M}\right)$. At the end of the simulation, performance are collected in order to calculate the value of the total cost incurred (block (7)). 


\subsection{Design of experiments and response surface methodology}

Two steps are necessary to optimize the control parameters of the four control policies: (a) determine the relationship between the performance criteria (i.e. incurred cost) and the independent variables of the model $\left(Z_{i}, a_{i}\right.$ and $\left.b_{i}, i \in\{1,2\}\right)$ and interactions which have a significant effect on the cost incurred, and (b) calculate the optimal parameter of the control policies which minimize the total cost incurred.

\subsection{Numerical example}

In order to significantly reduce the number of experiments without the loss of generality, the following assumptions are adopted:

- The setup times and the customer demand rates are considered constants;

- $\quad$ Both production and cost system parameters $\left(\mathrm{U}_{\mathrm{j}}^{\max }, \mathrm{d}_{\mathrm{i}}, \mathrm{T}_{\mathrm{ij}}^{\mathrm{s}}, \mathrm{c}_{\mathrm{i}}^{+}, \mathrm{c}_{\mathrm{i}}^{-}\right.$and $\left.\mathrm{c}_{\mathrm{ij}}^{\mathrm{s}}, \forall \mathrm{i}, \mathrm{j} \in\{1,2\}^{2}\right)$ are the same for the two products.

The first assumption implies that the value of the parameter $b_{i}, i \in\{1,2\}$ can be calculated by: $b_{i}=b=d * T_{s}$. This reduces the number of parameters to optimize. The second assumption implies that the thresholds values are equivalent for both product types $\left(\mathrm{Z}_{1}^{\mathrm{inv}}=\mathrm{Z}_{2}^{\mathrm{inv}}=\mathrm{Z}_{\mathrm{inv}}\right.$, $\mathrm{a}_{1}=\mathrm{a}_{2}=\mathrm{a}$ ). Given that $\mathrm{Z}_{\mathrm{PM}}<\mathrm{Z}_{1}^{\text {inv }}+\mathrm{Z}_{2}^{\text {inv }}=2 \mathrm{Z}_{\text {inv }}$ and $\mathrm{a} \leq \mathrm{Z}_{\text {inv }}$, two new variables $\alpha$ and $\beta$ are added to the simulation models in order to replace respectively the control parameters a and $\mathrm{Z}_{\mathrm{PM}}$ in the design of experiments. They are defined by:

$$
\begin{cases}\mathrm{a}=\alpha * \mathrm{Z}_{\mathrm{inv}}, & \alpha \in[0,1] \\ \mathrm{Z}_{\mathrm{PM}}=2 * \beta * \mathrm{Z}_{\mathrm{inv}}, & \beta \leq 1\end{cases}
$$

Note that when $\mathrm{Z}_{\mathrm{PM}} \ll 0$, all PM interventions are no longer ignored; we conclude that the PM strategy " $\mathrm{B}$ " is a specific case of the PM strategy "BJ" which is more general. Table 1 summarizes the system data which are inspired from the literature of the optimal control and maintenance strategies and could have been collected from real system. For example, the cost of negative inventory is much higher than the cost of positive inventory. As well, the machine parameter $U_{\max }$ must meet the feasibility requirement for the production system taking into account the customer demand. The time to failure $\left(\mathrm{T}_{\mathrm{f}}\right)$ and the durations of PM $\left(\mathrm{T}_{\mathrm{apm}}\right)$ and $\mathrm{CM}$ actions $\left(\mathrm{T}_{\mathrm{acm}}\right)$ could follow any probability distributions. From a practical point of view, 
historical data of failures and maintenance activities should be collected in order to define the probability distributions of $\left(\mathrm{T}_{\mathrm{f}}\right)\left(\mathrm{T}_{\mathrm{apm}}\right)$ and $\left(\mathrm{T}_{\mathrm{acm}}\right)$ (Rivera-Gomez et al., 2013).

Table 1. Simulation parameters.

\begin{tabular}{|c|c|c|c|c|c|c|c|c|c|c|c|}
\hline Parameters & $\mathrm{c}^{+}$ & $\mathrm{c}^{-}$ & $\mathrm{c}_{\mathrm{s}}$ & $\mathrm{c}_{\mathrm{cm}}$ & $\mathrm{c}_{\mathrm{pm}}$ & $\mathrm{T}_{\mathrm{s}}$ & $\mathrm{U}_{\mathrm{max}}$ & $\mathrm{d}$ & $\mathrm{T}_{\mathrm{f}}^{(1)}$ & $\mathrm{T}_{\mathrm{acm}}{ }^{(1)}$ & $\mathrm{T}_{\mathrm{apm}}{ }^{(1)}$ \\
\hline Values & 0.05 & 2 & 30 & 5000 & 2500 & 0.8 & 58 & 20 & $\begin{array}{c}\text { Log-N } \\
(120 ; 24)\end{array}$ & $\begin{array}{c}\text { Log-N } \\
(10 ; 1,6)\end{array}$ & $\begin{array}{c}\text { Log-N } \\
(7 ; 1)\end{array}$ \\
\hline \\
$\begin{array}{l}{ }^{(1)} T_{f}, T_{\text {apm }} \text { and } T_{\text {acm }} \text { define respectively the probability distributions of the time before failure and the } \\
\text { durations of PM and CM actions. }\end{array}$
\end{tabular}

For each combined control policy, a dependent variable (i.e. total cost) and several factors ( $\mathrm{T}_{\mathrm{PM}}$ and $\mathrm{Z}_{\mathrm{inv}}$ for HCP_B; $\mathrm{T}_{\mathrm{PM}}, \mathrm{Z}_{\mathrm{inv}}$ and a for IMHCP_B; $\mathrm{T}_{\mathrm{PM}}, \mathrm{Z}_{\mathrm{inv}}$ and $\mathrm{Z}_{\mathrm{PM}}$ for HCP_BJ and $\mathrm{T}_{\mathrm{PM}}, \mathrm{Z}_{\mathrm{inv}}, \mathrm{a}$ and $\mathrm{Z}_{\mathrm{PM}}$ for IMHCP_BJ) are considered. Based on the works of Gharbi et al. (2006), we assume that the value function (5) is convex. In this sense, given the small number of factors $(n \leq 4)$ for each joint control policy, we select full factorial designs at 3 levels $\left(3^{n}\right)$. These designs give more accurate results since each interaction is estimated separately. Five replicates were performed for each combination of factors which means a total of " 3 " $* 5$ " simulations according to the number of factors of the considered control policy. The simulation length is equal to $\mathrm{T}_{\infty}=500.000$ time units. This allows to reach the steady state, with a reasonable average run time equal to 0.2 minutes.

In order to study and understand the effects of independent variables $\left(\mathrm{T}_{\mathrm{PM}}, \mathrm{Z}_{\mathrm{inv}}, \alpha\right.$ and $\left.\beta\right)$ on the performance criteria (i.e.: total cost). A multi-factorial analysis of variance (ANOVA) and other statistical analyzes such as the calculation of the regression coefficients and the response surface generation were performed. The conformity of the model is also validated by conducting an analysis of the residual normality and of the homogeneity of variance. Using Statgraphics, Figure 5.a shows that the model representing IMHCP_BJ (see equation (11)) explains more than $96 \%$ of the observed variability in the total expected cost (Montgomery, 2008). In addition, all its main factors $\left(T_{P M}, Z_{i n v}, \alpha\right.$ and $\left.\beta\right)$, interactions and quadratic effects $\left(\mathrm{T}_{\mathrm{PM}}^{2}, \mathrm{Z}_{\mathrm{inv}}^{2}, \alpha^{2}\right.$ and $\left.\beta^{2}\right)$ except the interaction $\left(\mathrm{T}_{\mathrm{PM}} * \alpha\right)$, are significant at a $95 \%$ level of significance. 


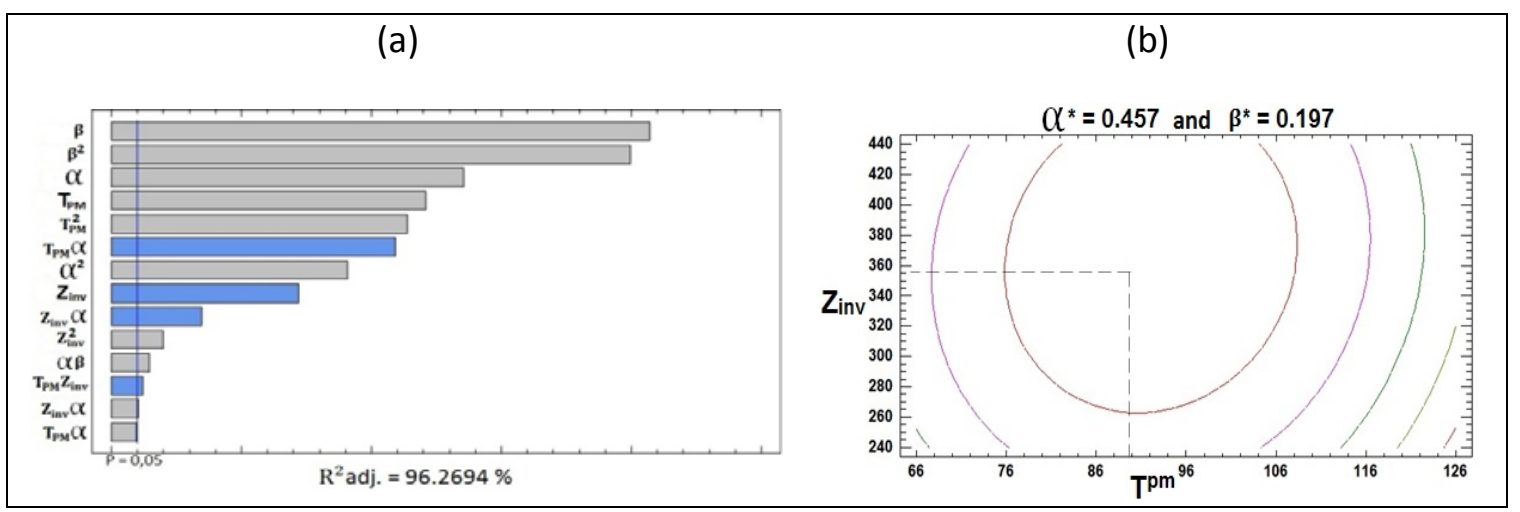

Figure 5. Pareto chart of dependent variables and response surface contour plot for IMHCP BJ.

The following equations represent the response surface function of the total cost for the different control policies.

$$
\begin{aligned}
& \mathrm{C}_{\text {HCP_B }}=229.712-3.00937 . T_{P M}-0.310629 . \mathrm{Z}_{\mathrm{inv}}-2.91424 .10^{-3} \cdot T_{P M} \cdot \mathrm{Z}_{\mathrm{inv}} \\
& +2.09215 \cdot 10^{-2} \cdot T_{P M}^{2}+1.1723 \cdot 10^{-3} \cdot Z_{\text {inv }}^{2} \\
& \mathrm{C}_{\mathrm{IMHCP} \_\mathrm{B}}=237.836-3.49926 . \mathrm{T}_{\mathrm{PM}}-0.0802741 . Z_{\mathrm{inv}}-45.9161 . \alpha-1.59369 .10^{-3} \cdot \mathrm{T}_{\mathrm{PM}} \cdot Z_{\mathrm{inv}} \\
& +0.144274 \cdot \mathrm{T}_{\mathrm{PM}} \cdot \alpha+2.20096 * 10^{-2} \cdot \mathrm{T}_{\mathrm{PM}}{ }^{2}+3.12284 \cdot 10^{-4} \cdot \mathrm{Z}_{\mathrm{inV}}^{2}+37.2982 \cdot \alpha^{2} \\
& \mathrm{C}_{\text {HCP_BJ }}=227.327-2.56585 . \mathrm{T}_{\mathrm{PM}}-0.481311 . Z_{\text {inv }}+24.1197 . \beta-6.95791 .10^{-4} \cdot \mathrm{T}_{\mathrm{PM}} \cdot \mathrm{Z}_{\mathrm{inv}} \\
& -0.275758 . \mathrm{T}_{\mathrm{PM}} \cdot \beta-0.0311542 . Z_{\mathrm{inv}} \cdot \beta+1.55096^{*} 10^{-2} \cdot \mathrm{T}_{\mathrm{PM}}{ }^{2}+1.17103 \cdot 10^{-3} \cdot Z_{\mathrm{inv}}^{2} \\
& +8.35226 . \beta^{2} \\
& \mathrm{C}_{\text {IMHCP_BJ }}=181.785-1.98293 . \mathrm{T}_{\mathrm{PM}}-0.188577 . \mathrm{Z}_{\text {inv }}-50.1811 . \alpha+32.2719 . \beta \\
& -3.46395 \cdot 10^{-4} \cdot \mathrm{T}_{\mathrm{PM}} \cdot \mathrm{Z}_{\mathrm{inv}}+0.422786 . \mathrm{T}_{\mathrm{PM}} \cdot \beta+0.020549 . \mathrm{Z}_{\mathrm{inv}} \cdot \alpha-0.0509712 . \mathrm{Z}_{\mathrm{inv}} \cdot \beta \\
& +3.86749 . \alpha \cdot \beta+1.22117 .10^{-2} \cdot \mathrm{T}_{\mathrm{PM}}{ }^{2}+3.0809 .10^{-4} \cdot \mathrm{Z}_{\mathrm{inv}}^{2}+46.0428 . \alpha^{2}+55.9828 . \beta^{2}
\end{aligned}
$$

A response surface methodology was subsequently carried out in order to optimize the total cost incurred (dependent variable) based on the significant variables. The response surface plot which corresponds to (11) is shown in Figure 5.

Table 2 presents the optimization results. It shows that for selected system parameters (see Table 1), the IMHCP_BJ gives the best result in terms of cost. In comparison with the HCP_B, the improvements resulting from HCP_BJ, IMHCP_B and IMHCP_BJ are respectively $2.61 \%, 8.34 \%$ and $14.60 \%$. These results demonstrate the advantage of combining the IMHCP with the "BJ". This avoids unnecessary setup operations and reduces 
the risk of shortages and the PM cost when preventive interventions follow immediately $\mathrm{CM}$ or setup actions. Table 2 confirms what was said in the introduction and shows that the integration of PM in the production planning yields significant cost benefits and increases the system availability. Note that a binary availability variable " $\mathrm{A}$ " is created using the simulation model. It is set to 1 if the machine is functional and 0 if the machine is down (under setup operations or repairs or PM actions). At the end of the simulation, the machine availability which represents the time-integral of " $\mathrm{A}$ " is then calculated.

Table 2. Optimization results.

\begin{tabular}{|c|l|c|c|c|c|c|c|}
\cline { 2 - 8 } \multicolumn{1}{c|}{} & \multicolumn{1}{c|}{ Policies } & $\mathrm{T}_{\mathrm{PM}}^{*}$ & $\mathrm{Z}_{\text {inv }}^{*}$ & $\mathrm{a}^{*}$ & $\mathrm{Z}_{\mathrm{PM}}^{*}$ & Total cost & Availability (\%) \\
\hline \multirow{2}{*}{$\begin{array}{c}\text { Without } \\
\text { PM }\end{array}$} & HCP & - & 291.53 & - & - & 75.06 & 91.02 \\
\cline { 2 - 8 } & IMHCP & - & 408.46 & 182.28 & - & 69.44 & 91.03 \\
\hline \multirow{4}{*}{$\begin{array}{c}\text { With } \\
\text { PM }\end{array}$} & HCP_B & 88.84 & 242.91 & - & - & 58.31 & 92.89 \\
\cline { 2 - 8 } & IMHCP_B & 91.13 & 361.08 & 158.63 & - & 53.82 & 92.88 \\
\cline { 2 - 8 } & HCP_BJ & 92.89 & 240.27 & - & 258.39 & 56.83 & 92.84 \\
\cline { 2 - 8 } & IMHCP_BJ & 89.68 & 357.53 & 163.33 & 141.14 & 50.88 & 92.83 \\
\hline
\end{tabular}

\section{Sensitivity analysis}

A sensitivity analysis is carried out with the goal of confirming the effectiveness and robustness of the resolution approach and studying the behavior of each joint control policies for different combinations of cost parameters (see Tables 3 and 4 - Scenarios 1-10). Tables 3 and 4 show that the optimal control parameters evolve in the same and right directions according to the variation of cost parameters. However, the effect of the setup cost depends on the Hedging Corridor Policy used (HCP or IMHCP) (see below, scenarios 5-6).

- Variation of the inventory cost (scenarios 1-4)

When $\mathrm{c}^{+}$increases, the $\mathrm{Z}_{\mathrm{inv}}^{*}$ and the $\mathrm{a}^{*}$ of all control policies decrease in order to avoid additional inventory costs. Therefore, the system when it faces a bigger risk of shortage reacts by decreasing the interval between two consecutive PM interventions $\left(\mathrm{T}_{\mathrm{PM}}^{*}\right)$ and the value of $\mathrm{Z}_{\mathrm{PM}}^{*}$ for the HCP_BJ and the IMHCP_BJ. Due to the increase of the cost of possession of finished products, the total cost of the four control policies increases. The opposite occurs when $\mathrm{c}^{+}$decreases. The $\mathrm{c}^{-}$has an inverse effect on the levels of buffer stocks $\left(\mathrm{Z}_{\mathrm{inv}}^{*}\right.$ and $\left.\mathrm{a}^{*}\right)$ 
compared to that of $\mathrm{c}^{+}$. In the same direction, the purpose of the increase of the PM frequency $\left(\mathrm{T}_{\mathrm{PM}}^{*}\right.$ and $\mathrm{Z}_{\mathrm{PM}}^{*}$ decrease) of all control policies is to reduce the number of failures.

- Variation of the setup cost (scenarios 5-6)

Tables 3 and 4 show that the influence of $\mathbf{c}_{\mathbf{s}}$ variation on the control parameters varies according to the adopted setup strategy (HCP or IMHCP). As regards the HCP_B and the HCP_BJ, the increase of $\mathbf{c}_{\mathbf{s}}$ gives preference to PM interventions in order to reduce the number of setup operations. Therefore, if $\mathrm{T}_{\mathrm{PM}}^{*}$ and $\mathrm{Z}_{\mathrm{PM}}^{*}$ related to the $\mathrm{HCP} B \mathrm{BJ}$ decreases, the number of setups also decreases. Furthermore, the increase of the number of PM interventions reduces the risk of failures, resulting in reduced buffer stocks ( $\mathrm{Z}_{\mathrm{inv}}^{*}$ and $\mathrm{a}^{*}$ decrease). In comparison with the HCP_B and the HCP_BJ, the effect of $\mathrm{c}_{\mathrm{s}}$ on the control parameters of the IMHCP_B and the IMHCP_BJ is the opposite. In fact, the number of setups is proportional to the value of $\mathrm{Z}_{\mathrm{inv}}^{*}$ and $\mathrm{a}^{*}$. Thus, when $\mathrm{c}_{\mathrm{s}}$ increases the values of $\mathrm{Z}_{\mathrm{inv}}^{*}$ and $\mathrm{a}^{*}$ increase in order to reduce the number of setups. Furthermore, due to the growth of buffer stocks, the risk of shortage decreases and the system reduces the PM interventions frequency $\left(\mathrm{T}_{\mathrm{PM}}{ }^{*}\right.$ and $\mathrm{Z}_{\mathrm{PM}}^{*}$ increase). The opposite occurs when $\mathrm{c}_{\mathrm{s}}$ decreases.

- Variation of the maintenance cost (scenarios 7-10)

When $\mathrm{c}_{\mathrm{pm}}$ increases, $\mathrm{T}_{\mathrm{PM}}^{*}$ and $\mathrm{Z}_{\mathrm{PM}}^{*}$ increase in order to avoid more expensive $\mathrm{PM}$ interventions. As a consequence, the risk of failures increases, hence the need to increase the value of $\mathrm{Z}_{\mathrm{inv}}^{*}$ and $\mathrm{a}^{*}$. The opposite occurs when $\mathrm{c}_{\mathrm{pm}}$ decreases. The variation of $\mathrm{c}_{\mathrm{cm}}$ has the opposite effect on the considered control policies when compared to that of $\mathrm{c}_{\mathrm{pm}}$.

This section shows that the IMHCP_BJ is the most advantageous in terms of cost. Its improvement compared to the IMHCP_B, the HCP_BJ and the HCP_B varies respectively from $4.74 \%$ to $7.67 \%$, from $9.48 \%$ to $14.6 \%$ and from $12.35 \%$ to $17.9 \%$. To conclude on this point, Tables 3 and 4 show that $\mathrm{C}_{\text {IMHCP_BJ }}^{*}<\mathrm{C}_{\text {IMHCP_B }}^{*}<\mathrm{C}_{\mathrm{HCP} \_\mathrm{BJ}}^{*}<\mathrm{C}_{\mathrm{HCP} \_\mathrm{B}}^{*}$. However, we believe that other comparative studies are necessary in order to draw meaningful conclusions and to determine if the above-mentioned inequality is always valid. 
Table 3. Sensitivity analysis table for control policies using the classical setup strategy (HCP).

\begin{tabular}{|c|c|c|c|c|c|c|c|c|c|c|c|c|c|}
\hline \multirow{2}{*}{ Scenario } & \multicolumn{5}{|c|}{ Cost parameters } & \multicolumn{2}{|c|}{$T_{P M}^{*}$} & \multicolumn{2}{|c|}{$Z_{i n v}^{*}$} & \multirow{2}{*}{$\begin{array}{c}Z_{P M}^{*} \\
\text { HCP_BJ }\end{array}$} & \multicolumn{2}{|c|}{ Total cost $\left(C^{*}\right)$} & \multirow{2}{*}{ Remark } \\
\hline & $c^{+}$ & $c^{-}$ & $c_{S}$ & $c_{p m}$ & $c_{c m}$ & HCP_B & $\mathrm{HCP} \_\mathrm{BJ}$ & HCP_B & $\mathrm{HCP}_{-} \mathrm{BJ}$ & & HCP_B & HCP_BJ & \\
\hline Basic & 0,05 & 2 & 30 & 2500 & 5000 & 88,84 & 92,89 & 242,91 & 240,27 & 258,39 & 58,31 & 56,83 & se \\
\hline 1 & 0,03 & 2 & 30 & 2500 & 5000 & 90,20 & 93,87 & 261,40 & 258,11 & 305,05 & 50,59 & 49,18 & $T_{P M}^{*} \uparrow, Z_{i n v}^{*} \uparrow, Z_{P M}^{*} \uparrow, C^{*} \downarrow$ \\
\hline 2 & 07 & 2 & 30 & 2500 & 5000 & 87,42 & 1,86 & 224,67 & 222,77 & 216,27 & 1 & 63,78 & $T_{P M}^{*} \downarrow, Z_{i n v}^{*} \downarrow, Z_{P M}^{*} \downarrow, C^{*} \uparrow$ \\
\hline 3 & 0,05 & 1,8 & 30 & 2500 & 5000 & 88,84 & 92,96 & 238,29 & 235,62 & 249,76 & 58,09 & 56,67 & $T_{P M}^{*} \uparrow, Z_{i n v}^{*} \downarrow, Z_{P M}^{*} \downarrow, C^{*} \downarrow$ \\
\hline 4 & 0,05 & 2,2 & 30 & 2500 & 5000 & 88,82 & 92,82 & 246,75 & 244,09 & 265,26 & 58,49 & 56,94 & $T_{P M}^{*} \downarrow, Z_{i n v}^{*} \uparrow, Z_{P M}^{*} \uparrow, C^{*} \uparrow$ \\
\hline 5 & 0,05 & 2 & 26 & 2500 & 5000 & 88,92 & 93,00 & 243,00 & 240,33 & 259,65 & 57,15 & 55,65 & $T_{P M}^{*} \uparrow, Z_{i n v}^{*} \uparrow, Z_{P M}^{*} \uparrow, C^{*} \downarrow$ \\
\hline 6 & 0,05 & 2 & 34 & 2500 & 5000 & 88,76 & 92,77 & 242,83 & 240,21 & 256,95 & 59,47 & 58,00 & $T_{P M}^{*} \downarrow, Z_{i n v}^{*} \downarrow, Z_{P M}^{*} \downarrow, C^{*} \uparrow$ \\
\hline 7 & 0,05 & 2 & 30 & 2000 & 5000 & 86,69 & 89,24 & 240,22 & 238,49 & 214,63 & 52,78 & 51,58 & $T_{P M}^{*} \downarrow, Z_{i n v}^{*} \downarrow, Z_{P M}^{*} \downarrow, C^{*} \downarrow$ \\
\hline 8 & 0,05 & 2 & 30 & 3000 & 5000 & 90,88 & 96,85 & 245,48 & 242,16 & 314,10 & 63,67 & 61,68 & $T_{P M}^{*} \uparrow, Z_{i n v}^{*} \uparrow, Z_{P M}^{*} \uparrow, C^{*} \uparrow$ \\
\hline 9 & 0,05 & 2 & 30 & 2500 & 4000 & 90,25 & 96,54 & 244,74 & 241,85 & 309,41 & 57,86 & 56,05 & $T_{P M}^{*} \uparrow, Z_{i n v}^{*} \uparrow, Z_{P M}^{*} \uparrow, C^{*} \downarrow$ \\
\hline 10 & 0,05 & 2 & 30 & 2500 & 6000 & 87,64 & 90,37 & 241,36 & 239,16 & 225,28 & 58,70 & 57,35 & $T_{P M}^{*} \downarrow, Z_{i n v}^{*} \downarrow, Z_{P M}^{*} \downarrow, C^{*} \uparrow$ \\
\hline
\end{tabular}

Table 4. Sensitivity analysis table for control policies using the modified setup strategy (IMHCP).

\begin{tabular}{|c|c|c|c|c|c|c|c|c|c|c|}
\hline \multirow{2}{*}{ Scenario } & \multicolumn{2}{|c|}{$T_{P M}^{*}$} & \multicolumn{2}{|c|}{$Z_{i n v}^{*}$} & \multicolumn{2}{|c|}{$a^{*}$} & \multirow{2}{*}{$\frac{Z_{P M}^{*}}{\mathrm{IMHCP} \text { BJ }}$} & \multicolumn{2}{|c|}{ Total cost $\left(C^{*}\right)$} & \multirow{2}{*}{ Remark } \\
\hline & IMHCP_B & IMHCP_BJ & IMHCP_B & IMHCP_BJ & IMHCP_B & IMHCP_BJ & & IMHCP_B & IMHCP_BJ & \\
\hline Basic & 91,13 & 89,68 & 361,08 & 357,53 & 158,63 & 163,33 & 141,14 & 53,82 & 50,88 & Basic case \\
\hline 1 & 92,43 & 89,94 & 395,30 & 366,00 & 173,82 & 166,81 & 148,00 & 45,48 & 42,91 & $T_{P M}^{*} \uparrow, Z_{i n \vartheta}^{*} \uparrow, a^{*} \uparrow, Z_{P M}^{*} \uparrow, C^{*} \downarrow$ \\
\hline 2 & 89,43 & 88,54 & 317,33 & 314,19 & 139,64 & 146,07 & 108,56 & 61,12 & 58,13 & $T_{P M}^{*} \downarrow, Z_{i n v}^{*} \downarrow, a^{*} \downarrow, Z_{P M}^{*} \downarrow, C^{*} \uparrow$ \\
\hline 3 & 91,23 & 89,82 & 350,92 & 346,74 & 154,21 & 159,32 & 132,61 & 53,47 & 50,80 & $T_{P M}^{*} \uparrow, Z_{i n v}^{*} \downarrow, a^{*} \downarrow, Z_{P M}^{*} \downarrow, C^{*} \downarrow$ \\
\hline 4 & 91,01 & 89,55 & 369,48 & 366,00 & 162,26 & 166,45 & 148,28 & 54,13 & 50,91 & $T_{P M}^{*} \downarrow, Z_{i n v}^{*} \uparrow, a^{*} \uparrow, Z_{P M}^{*} \uparrow, C^{*} \uparrow$ \\
\hline 5 & 91,08 & 89,65 & 359,69 & 356,23 & 157,74 & 162,69 & 140,13 & 53,57 & 50,63 & $T_{P M}^{*} \downarrow, Z_{i n v}^{*} \downarrow, a^{*} \downarrow, Z_{P M}^{*} \downarrow, C^{*} \downarrow$ \\
\hline 6 & 91,17 & 89,71 & 362,36 & 358,80 & 159,43 & 163,98 & 142,10 & 54,08 & 51,14 & $T_{P M}^{*} \uparrow, Z_{i n v}^{*} \uparrow, a^{*} \uparrow, Z_{P M}^{*} \uparrow, C^{*} \uparrow$ \\
\hline 7 & 89,11 & 85,99 & 355,96 & 352,19 & 157,71 & 162,04 & 110,53 & 48,49 & 45,03 & $T_{P M}^{*} \downarrow, Z_{i n v}^{*} \downarrow, a^{*} \downarrow, Z_{P M}^{*} \downarrow, C^{*} \downarrow$ \\
\hline 8 & 93,03 & 93,19 & 365,95 & 363,83 & 159,45 & 164,80 & 183,52 & 59,00 & 56,34 & $T_{P M}^{*} \uparrow, Z_{i n v}^{*} \uparrow, a^{*} \uparrow, Z_{P M}^{*} \uparrow, C^{*} \uparrow$ \\
\hline 9 & 92,68 & 92,91 & 365,02 & 364,61 & 159,33 & 164,97 & 191,02 & 53,23 & 50,55 & $T_{P M}^{*} \uparrow, Z_{i n v}^{*} \uparrow, a^{*} \uparrow, Z_{P M}^{*} \uparrow, C^{*} \downarrow$ \\
\hline 10 & 89,80 & 87,13 & 357,67 & 353,10 & 157,93 & 162,28 & 113,49 & 54,34 & 50,89 & $T_{P M}^{*} \downarrow, Z_{i n v}^{*} \downarrow, a^{*} \downarrow, Z_{P M}^{*} \downarrow, C^{*} \uparrow$ \\
\hline
\end{tabular}




\section{Comparison of the joint control policies for a wide range system configurations}

In this section, a further study of the influence of the different cost and time system parameters on the considered control policies is conducted. The way that the proposed control policies evolve in relation to one another is also highlighted. Every time a system parameter is considered, the others remain fixed as defined in the basic scenario (Table 1).

\subsection{Variation of the cost parameters}

Figure 6 confirms that for the different cost parameters adopted, the total cost corresponding to the IMHCP_BJ is always less than that of other control policies. However, it also shows that unlike the sensitivity analysis (Section 4), the total cost of the HCP_BJ may fall below that of the IMHCP_B (see Figures 6.d and 6.e). Indeed, with the increase of $c_{p m}$ (Figure 6.d), jumps when performing PM interventions become more significant and PM actions are less attractive since they cost more. This explains why at some level $\left(c_{\mathrm{pm}}>4500\right)$ the total cost of the IMHCP_B becomes higher than that of the HCP_BJ which take advantage of its capability to jump PM interventions (see equation (6)). The same phenomenon is observed when $\mathrm{C}_{\mathrm{s}}$ drops below 17 (Figure 6.e). Indeed, in response to the decrease of the setup costs for HCP_BJ, the advantage of the IMHCP_B which avoids several unnecessary setup operations becomes less attractive. Furthermore, the HCP_BJ continue to benefit from the PM jumps when the buffer stocks level is considered insufficient in comparison with the IMHCP_B. Note that the growth of the $\mathrm{c}_{\mathrm{s}}$ value remarkably increases the " $\mathrm{C}_{\mathrm{HCP} \_}^{*} \mathrm{~B}-\mathrm{C}_{\mathrm{IMHCP} \_\mathrm{B}}^{*}$ " and the " $\mathrm{C}_{\mathrm{HCP} \_ \text {BJ }}^{*}-\mathrm{C}_{\text {IMHCP_BJ }}^{*}$ ". This is essentially due to the high number of setup operations executed by HCP_B and HCP_BJ, which switch the production from one product type to another, as soon as an inventory level $\left(\mathrm{x}_{\mathrm{i}}, \mathrm{i} \in\{1,2\}\right)$ reaches its threshold $\left(\mathrm{Z}_{\text {inv }}\right)$.

\subsection{Variation of the time parameters}

The objective of this section is to study the effect of the mean corrective and preventive maintenance times (MTCM and MTPM) and their standard deviation (SDCM and SDPM) as well as the setup time $\left(\mathrm{T}_{\mathrm{S}}\right)$. The results obtained are presented in Figure 7 . They demonstrate once again the economic superiority of the IMHCP_BJ. 


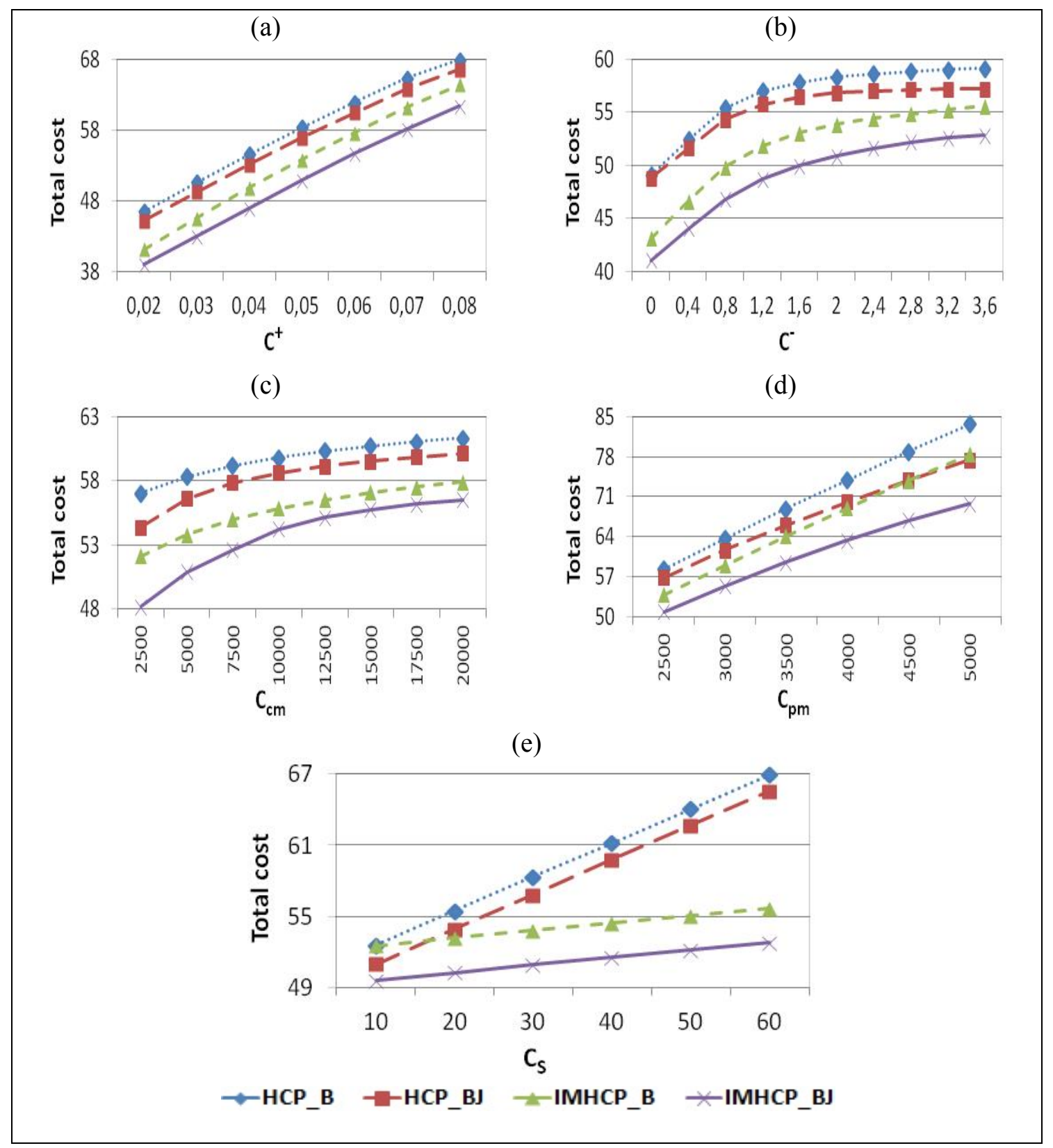

Figure 6. Effect of cost system parameters on the incurred total cost of the control policies.

As presented in Section 5.1, the value of the total cost of the IMHCP_B could exceed that of the HCP_BJ (see Figure 7.e). Unlike the base scenario $\left(\mathrm{T}_{\mathrm{S}}=0.8\right)$, the excess of the total cost generated by IMHCP_B over that generated by HCP_BJ when $\mathrm{T}_{\mathrm{S}}$ increases $\left(\mathrm{T}_{\mathrm{S}}>1.25\right)$, is explained by three main events: the growth of inventory costs for the control policy using the IMHCP_B in order to avoid longer setup times involving an important risk of shortages, the economic advantage of the jumps of PM interventions (for HCP_BJ) and the remarkable 
decrease of the number of setups; and then setup costs for the control policy using a classical setup policy (HCP_BJ). Note that the IMHCP generates a greater risk of shortages during the downtime than the HCP which use the maximal production rate throughout the availability period of the machine. It is interesting to note that the decrease of the total cost incurred for HCP_B and HCP_BJ presented in Figure 7.e is mainly due to the considerable reduction of the number of performed setup operations. Indeed, these control policies generate less setups when $\mathrm{T}_{\mathrm{S}}$ increase.

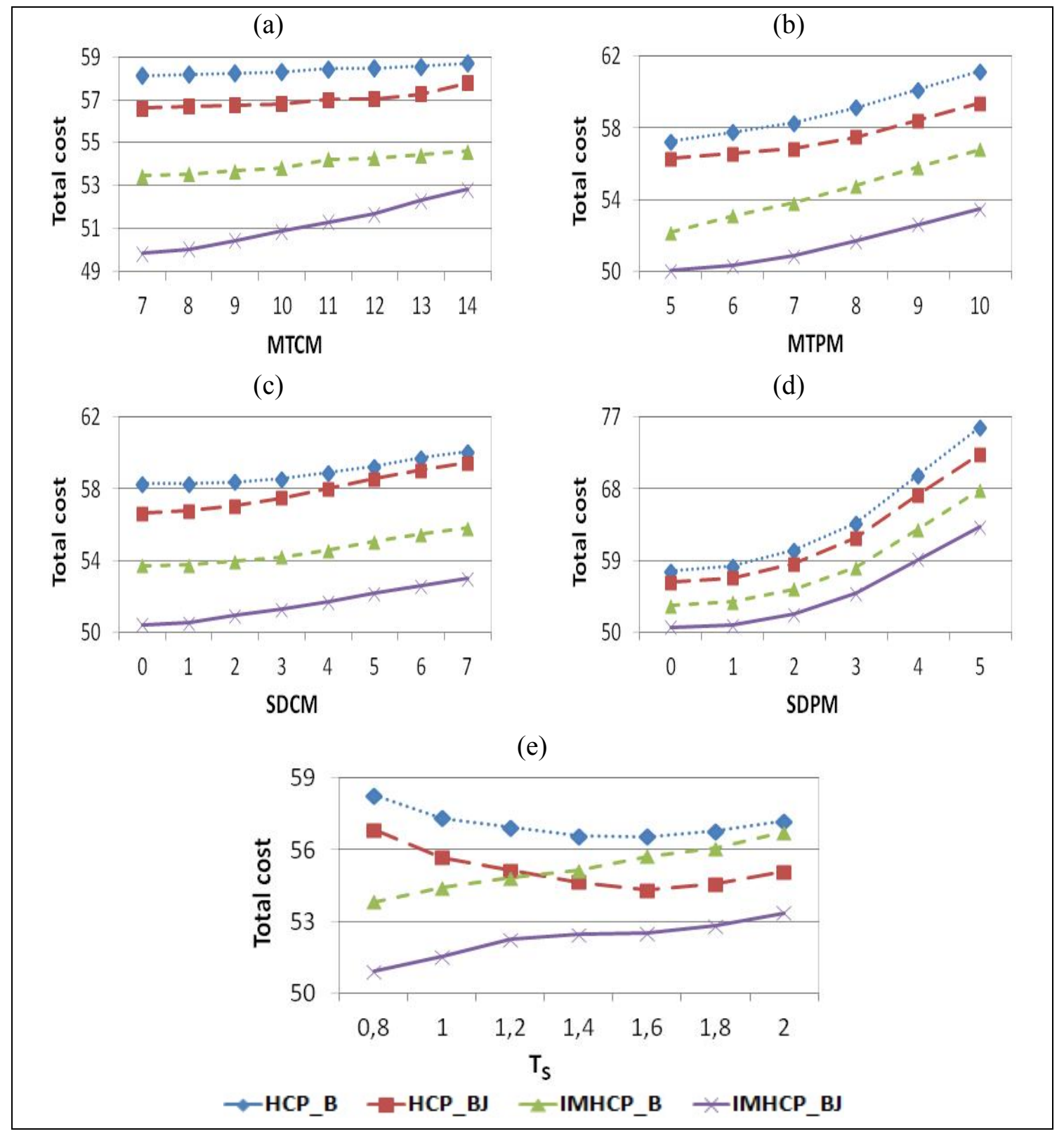

Figure 7. Effect of time system parameters on the incurred total cost of the control policies. 
This study also demonstrates that the increase of MTCM (Figure 7.a) allows the production system to perform more $\mathrm{PM}$ interventions $\left(\mathrm{Z}_{\mathrm{PM}}^{*}\right.$ and $\mathrm{T}_{\mathrm{PM}}^{*}$ decrease) in order to reduce the number of failures and shortages. Thus, the value of " $\mathrm{C}_{\mathrm{HCP} \mathrm{B}_{\mathrm{B}}}^{*}-\mathrm{C}_{\mathrm{HCP} \_\mathrm{BJ}}^{*}$ " and " $\mathrm{C}_{\mathrm{IMHCP} B}^{*} \mathrm{~B}-\mathrm{C}_{\mathrm{IMHCP} \_\mathrm{B}}^{*}$ " decreases, since skipping a PM intervention further increases the risk of failures. Figure 7.b shows that the jumps when performing PM interventions (for HCP_BJ and IMHCP_BJ) become more interesting ( $\mathrm{Z}_{\mathrm{PM}}^{*}$ and $\mathrm{T}_{\mathrm{PM}}^{*}$ increase) when the MTPM

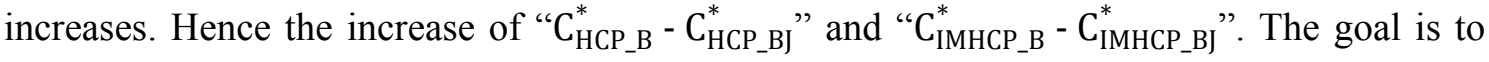
avoid additional PM costs but also shortage costs (PM time is longer). Note that the considered control policies evolve in the same directions as the MTCM and MTPM presented in the previous paragraph, in response to variations in the standard deviations SDCM (Figure 7.c) and SDPM (Figure 7.d) respectively.

In summary, the comparison of the considered control policies in terms of cost shows that for a wide range of system configurations, the IMHCP_BJ gives a better result than the other control policies. This advantage is due to its capacity to avoid a high number of unnecessary setup operations and PM interventions.

\section{Conclusion}

A joint production, setup and maintenance control problem of an unreliable and a non-flexible manufacturing system producing two products has been addressed here. The primary objective is to propose a proper structure of a control policy which can combine simultaneously the production, the setup and the maintenance activities. Four combined policies have been presented. In order to control the production rate and the sequence of setup operations, the Hedging Corridor Policy (HCP) and the Improved Modified Hedging Corridor Policy (IMHCP) were used. Two periodic PM strategies are also introduced in this work. The first one is the classical Block Replacement Policy and the second one is characterized by preventive interventions which can be skipped if the inventories level is considered insufficient. Four combined discrete-continuous simulation models are developed in order to represent the different control policies considered. An experimental resolution approach which integrates the simulation with the design of experiments, the analysis of variance and the response surface methodology was used in order to determine the optimal solutions for each combined control policy. A sensitivity analysis and a comparative study were also 
conducted confirming the robustness of the approach used and the effectiveness of the considered control policies.

This work studies the effect of a wide range of cost and time variables of the system on the optimal control parameters and the incurred total cost of the considered policies. The main conclusion is that the IMHCP_BJ which combines the IMHCP with the Block Replacement Policy with jumps gives the best results in terms of cost. Its advantage is to avoid unnecessary setup operations and to reduce the risk of shortages and the waste of new components when PM interventions follow immediately CM or setup actions. Further extensions of this work can be studied by considering others PM strategies and other structure of the production and the setup control policies.

\section{References}

Akella, R., \& Kumar, P.R., (1986). Optimal Control of Production Rate in a Failure Prone Manufacturing System. IEEE Transactions on Automatic Control, 31(2), pp. 116-126.

Aramon Bajestani, M., Banjevica, D., \& Becka J.C., (2014). Integrated maintenance planning and production scheduling with Markovian deteriorating machine conditions. International Journal of Production Research, 52(24). DOI:10.1080/00207543.2014.931609.

Assid, M., Gharbi, A., \& Dhouib, K. (2014a). Joint production and subcontracting planning of unreliable multi-facility multi-product production systems. Omega, 50, pp. 54-69.

Assid, M., Gharbi, A., \& Hajji, A,. (2014b). Joint production and setup control policies: an extensive study addressing implementation issues via quantitative and qualitative criteria. The International Journal of Advanced Manufacturing Technology. 72(5-8), pp. 809-826.

Ayed, S., Dellagi, S., Rezg, N., (2012). Joint optimisation of maintenance and production policies considering random demand and variable production rate. International Journal of Production Research, 50(23), pp. 6870-6885.

Bai, S.X., \& Elhafsi, M., (1997). Scheduling of an unreliable manufacturing system with nonresumable set-ups. Computers Ind. Eng., 32, pp. 909-925.

Berthaut, F., Gharbi, A., \& Dhouib, K. (2011). Joint modified block replacement and production/inventory control policy for a failure-prone manufacturing cell. Omega, 39(1), pp. 642-654.

Cassady, C.R., \& Kutanoglu, E., (2005). Integrating preventive maintenance planning and production scheduling for a single machine. IEEE Transaction on Reliability, 54(2), pp. 304-309.

Dhouib, K., Gharbi, A., Ben Aziza, M.N., 2012, Joint optimal production control/preventive maintenance policy for imperfect process manufacturing cell. International Journal of Production Economics, 137, pp. 126-136. 
Gharbi, A., \& Kenné, J. P., (2000). Production and preventive maintenance rates control for a manufacturing system: An experimental design approach. International Journal of Production Economics, 65(3), pp. 275-287.

Gharbi, A., Kenné, J.P., \& Beit, M., (2007). Optimal safety stocks and preventive maintenance periods in unreliable manufacturing systems. International Journal of Production Economics, 107(2), pp. 422-434.

Gharbi, A., Hajji, A., \& Dhouib, K., (2011). Production rate control of an unreliable manufacturing cell with adjustable capacity. International Journal of Production Research, 49(21), pp. 6539-6557.

Gharbi, A., Kenné, J. P., \& Hajji, A., (2006). Operational level-based policies in production rate control of unreliable manufacturing systems with set-ups. International Journal of Production Research, 44(3), pp. 545-567.

Hajji, A., Gharbi, A., Kenné, J.P., \& Hidehiko, Y. (2009). "Production and Changeover Control Policies of a Class of Failure Prone Buffered Flow-shops." Production Planning \& Control, 20(8), pp. 785-800.

Kenné, J.P., and Gharbi, A. (2000). Production planning problem in manufacturing systems with general failure and repair time distributions. Production Planning and Control, 11, pp. 581-588.

Kuo, Y., \& Chang, ZA., (2007). Integrated production scheduling and preventive maintenance planning for a single machine under a cumulative damage failure process. Naval Research Logistics, 54(6), pp. 602-614.

Montgomery, D.C., (2008). Design and Analysis of Experiments, 7th edition, New York: John Wiley \& Sons.

Reineke, D.M., Murdock, W.P.J., Pohl, E.A., \& Rehmert, I., (1999). Improving availability and cost performance for complex systems with preventive maintenance. Proceedings of the Annual Reliability and Maintainability Symposium, pp. 383-388.

Rezg, N., Dellagi, S., Chelbi, A., (2008). Joint optimal control and preventive maintenance policy. International Journal of Production Research, 46(19), pp. 5349-5365.

Rivera-Gomez, H., Gharbi, A., \& Kenné, J. P. (2013). Joint control of production, overhaul, and preventive maintenance for a production system subject to quality and reliability deteriorations. International journal, advanced manufacturing technology, 69(9-12), 21112130.

Wang, H., (2002). A survey of maintenance policies of deteriorating systems. European Journal of Operational Research, 139, pp. 469-489.

Wang, S., \& Liu, M., (2013). A branch and bound algorithm for single-machine production scheduling integrated with preventive maintenance planning. International Journal of Production Research, 51(3), pp. 847-868.

Wee, H.M., \& Widyadana, G.A., (2013). A production model for deteriorating items with stochastic preventive maintenance time and rework process with FIFO rule. Omega, 41, pp. 941-954. 